\title{
Calibration of Magnetic Field Sensors with two mass-market GNSS receivers
}

\author{
Patrick Henkel $(*, * *)$, Philipp Berthold $(* *)$ and Jane Jean Kiam (**) \\ * Technische Universität München, Munich, Germany \\ ** Advanced Navigation Solutions - ANavS, Munich, Germany \\ patrick.henkel@tum.de,philipp.berthold@anavs.de,jane.kiam@anavs.de
}

\begin{abstract}
Global Navigation Satellite System (GNSS) signals, inertial measurements (angular rates, accelerations) and magnetometer measurements complement each other for position determination: GNSS provides a precise and drift-free position solution but is susceptible to signal outages. Inertial measurements are continuously available and of higher rate but suffer from integration drifts. Magnetic field measurements provide an instantaneous orientation in static conditions but are affected by both static and dynamic disturbances.

In this paper, we provide a calibration method for magnetometers, which determines the biases and misalignment errors of the magnetometer as well as the magnetic flux including static disturbances. The method uses the iterative Gauss-Newton method and precise attitude information (heading, pitch) obtained from two low-cost GPS receivers. The attitude determination requires a tree search of the carrier phase integer ambiguities using a priori information on the distance between both GPS receivers.

We also verified the proposed method with kinematic measurements from the CMPS10 sensor. We observe an accuracy of a few degrees for the unfiltered heading and a heading offset of less than $10^{\circ}$ in $99.5 \%$ of all measurement epochs.
\end{abstract}

Index Terms-Magnetic field sensor, Attitude determination, Integer Ambiguity Resolution, Calibration.

\section{INTRODUCTION}

GPS receivers can track the carrier phase of GPS signals with millimeter to centimeter-level accuracy. Low-cost GPS receivers are becoming attractive in numerous applications. We focus on attitude determination for future driving assistance systems. As GPS signals are weak and easily blocked by shadowing, additional sensors are required to provide a continuous solution. Inertial sensors are attractive to support the GPS solution, i.e. they can be used to coast the GPS solution during short signal outages (e.g. passing below bridges or trees), to detect and correct cycle slips, to accelerate the ambiguity re-fixing after short signal outages, and to increase the data rate. However, inertial sensors are not beneficial for integer ambiguity resolution of standing vehicles. This initial integer ambiguity resolution can last a minute for low-cost GPS receivers as code multipath is often in the order of several tens of metres. Magnetometers can provide an initial orientation of the vehicle and, thereby, substantially shorten the time of initial GPS carrier phase integer ambiguity resolution.

However, magnetometers need an initial calibration. Crassidis et al. [1] and Psiaki et al. [2] used extended and unscented Kalman filters to estimate the sensor biases and scaling factors.
In this paper, we provide a calibration method which uses precise GPS-based attitude information to determine misalignment errors of the magnetometer, biases of the magnetic flux measurements, and the magnetic flux including static disturbances.

We also apply our method to three-dimensional magnetic flux measurements of a low-cost CMPS10 sensor and two lowcost u-blox LEA 6T GPS receivers, that were mounted on the roof of a vehicle during a test drive. We observe an error of only a few degrees for the calibrated magnetic heading, which is provided with a measurement rate of $100 \mathrm{~Hz}$.

\section{Measurement Models}

In this section, we introduce measurement models for the magnetometer and for the double difference code and carrier phase measurements of two low-cost GPS receivers.

\section{A. Measurement model for magnetic field sensor}

We use the CMPS10 sensor of Devantech, which provides three-dimensional magnetic flux measurements as raw data. We model these magnetic flux measurements in the sensorfixed (s-) frame (centered in the sensor and aligned with the principal axes of the sensor) at time $t_{i}$ as

$$
\begin{aligned}
\left(\begin{array}{c}
m_{x}^{\mathrm{s}}\left(t_{i}\right) \\
m_{y}^{\mathrm{s}}\left(t_{i}\right) \\
m_{z}^{\mathrm{s}}\left(t_{i}\right)
\end{array}\right)= & \Lambda R_{\mathrm{n}}^{\mathrm{s}}\left(\varphi\left(t_{i}\right)+\Delta \varphi, \theta\left(t_{i}\right)+\Delta \theta, \psi\left(t_{i}\right)+\Delta \psi\right) \\
& \cdot m^{\mathrm{n}}\left(t_{i}\right)+b^{\mathrm{s}}+\eta_{m}\left(t_{i}\right)
\end{aligned}
$$

with the scaling factor matrix $\Lambda$, the rotation matrix $R_{\mathrm{n}}^{\mathrm{s}}$ from the navigation (n-) frame (centered in the sensor and aligned with the East, North and Up-axis) into the s-frame, the roll, pitch and heading angles $\{\varphi, \theta, \psi\}$ of the vehicle, the misalignment errors $\{\Delta \varphi, \Delta \theta, \Delta \psi\}$, the static disturbances/ biases $b_{\mathrm{s}}$ of the magnetic field measurements, and the noise $\eta_{m}$ including dynamic disturbances.

As the magnetic flux mainly points in Northern direction (the magnetic declination is assigned to the misalignment error) and as the scaling factor is negligible for most magnetic field sensors, the general model of Eq. (1) simplifies. Furthermore, the roll angle is negligible in automotive applications such that Eq. (1) becomes

$$
\begin{aligned}
m_{x}^{\mathrm{s}}\left(t_{i}\right)= & \cos \left(\theta\left(t_{i}\right)+\Delta \theta\right) \cdot \sin \left(\psi\left(t_{i}\right)+\Delta \psi\right) \cdot m_{\mathrm{N}}\left(t_{i}\right) \\
& +b_{x}+\eta_{m_{x}}\left(t_{i}\right) \\
m_{y}^{\mathrm{s}}\left(t_{i}\right)= & \cos \left(\psi\left(t_{i}\right)+\Delta \psi\right) \cdot m_{\mathrm{N}}\left(t_{i}\right)+b_{y}+\eta_{m_{y}}\left(t_{i}\right)
\end{aligned}
$$


and

$$
\begin{aligned}
m_{z}^{\mathrm{s}}\left(t_{i}\right)= & \sin \left(\theta\left(t_{i}\right)+\Delta \theta\right) \cdot \sin \left(\psi\left(t_{i}\right)+\Delta \psi\right) \cdot m_{\mathrm{N}}\left(t_{i}\right) \\
& +b_{z}+\eta_{m_{z}}\left(t_{i}\right),
\end{aligned}
$$

which leaves the attitude $\{\psi, \theta\}$, the attitude misalignment errors $\{\Delta \psi, \Delta \theta\}$, the biases $\left\{b_{x}, b_{y}, b_{z}\right\}$, and the magnitude of the magnetic flux $m_{\mathrm{N}}\left(t_{i}\right)$ in northern direction as unknowns.

\section{B. Measurement model for GPS measurements}

In this subsection, we describe our model for double difference (DD) measurements of two low-cost GPS receivers based on [3] and [4]. The model needs to take the particularities of these receivers into account, i.e. the lack of synchronization, code multipath and $\lambda / 2$ cycle slips [5]. We model the double difference carrier phase measurement for the receivers $\{1,2\}$ and satellites $\{k, l\}$ as

$$
\begin{aligned}
\varphi_{12}^{k l}\left(t_{i}\right)= & \left(\vec{e}^{k l}\left(t_{i}\right)\right)^{\mathrm{T}} \vec{b}_{12}\left(t_{i}\right)+c_{12}^{k l}\left(t_{i}\right) \\
& +\lambda N_{12}^{k l}+\lambda / 2 \Delta N_{12}^{k l}\left(t_{i}\right)+\varepsilon_{\varphi_{12}^{k l}}\left(t_{i}\right),
\end{aligned}
$$

with the satellite-satellite single difference $\vec{e}^{k l}=\vec{e}^{k}-\vec{e}^{l}$ of the satellite-receiver line of sight vectors, the unknown baseline vector $\vec{b}_{12}$ between two GPS receivers, the synchronization correction $c_{12}^{k l}$ (correcting for the receiver and satellite movements within the differential receiver clock offset), the wavelength $\lambda$, the unknown DD integer ambiguity $N_{12}^{k l} \in \mathbb{Z}$, the unknown DD cycle slip $\Delta N_{12}^{k l} \in \mathbb{Z}$ and the DD phase noise including multipath. We use a similar model for the DD pseudorange measurements, i.e.

$\rho_{12}^{k l}\left(t_{i}\right)=\vec{e}^{k l}\left(t_{i}\right) \vec{b}_{12}\left(t_{i}\right)+c_{12}^{k l}\left(t_{i}\right)+\Delta \rho_{\mathrm{MP}, 12}^{k l}\left(t_{i}\right)+\varepsilon_{\rho_{12}^{k l}}\left(t_{i}\right)$,

with $\Delta \rho_{\mathrm{MP}, 12}^{k l}$ being the unknown DD code multipath and $\varepsilon_{\rho_{12}^{k l}}$ denoting the DD code noise.

As both GPS receivers are mounted on the roof of a vehicle, the length $l_{12}=\left\|\vec{b}_{12}\right\|$ can be assumed to be constant. We assume that there is a baseline length measurement available, which we model as

$$
\bar{l}_{12}=\left\|\vec{b}_{12}\left(t_{i}\right)\right\|+\varepsilon_{\bar{l}_{12}},
$$

with $\varepsilon_{\bar{l}_{12}} \sim \mathcal{N}\left(0, \sigma_{\bar{l}_{12}}^{2}\right)$ describing the error of the baseline length measurement.

\section{INTEGER AMBIGUITY RESOLUTION WITH LOW-COST GPS RECEIVERS}

In this section, we determine the DD carrier phase ambiguities by integer least-squares estimation using baseline length a priori information. A static baseline is considered and DD phase and code measurements from $n=800$ epochs are used to achieve sufficient redundancy. The baseline vector $\vec{b}_{12}$ and ambiguities $N_{12}$ are determined by minimizing the weighted sum of squared measurement residuals and of squared baseline length residuals, i.e.

$\min _{\vec{b}_{12}, N_{12}}\left(\left\|\Psi_{12}-H \vec{b}_{12}-A N_{12}\right\|_{\Sigma_{\Psi_{12}}^{-1}}^{2}+\left(\left\|\vec{b}_{12}\right\|-\bar{l}_{12}\right)^{2} / \sigma_{\bar{l}_{12}}^{2}\right)$ with the DD carrier phase and pseudorange measurements $\Psi_{12}=\left(\lambda \varphi_{12}^{\mathrm{T}}, \rho_{12}^{\mathrm{T}}\right)^{\mathrm{T}}$ of $n$ epochs, i.e. the carrier phases $\lambda \varphi_{12}=\left(\lambda \varphi_{12}^{\mathrm{T}}\left(t_{1}\right), \ldots, \lambda \varphi_{12}^{\mathrm{T}}\left(t_{n}\right)\right)^{\mathrm{T}}$ and $\rho_{12}=$ $\left(\rho_{12}^{\mathrm{T}}\left(t_{1}\right), \ldots, \rho_{12}^{\mathrm{T}}\left(t_{n}\right)\right)^{\mathrm{T}}$, the DD integer ambiguities $N_{12}$ from all available DD with a common reference satellite, and the mapping matrices $H$ and $A$. We apply a DD synchronization correction to restore the integer property of DD ambiguities, i.e.

$$
\begin{aligned}
\lambda \varphi_{12}\left(t_{i}\right) & =\left(\begin{array}{c}
\lambda \varphi_{12}^{12}\left(t_{i}\right)-c_{12}^{12}\left(t_{i}\right) \\
\vdots \\
\lambda \varphi_{12}^{1 K}\left(t_{i}\right)-c_{12}^{1 K}\left(t_{i}\right)
\end{array}\right) \\
\rho_{12}\left(t_{i}\right)= & \left(\begin{array}{c}
\rho_{12}^{12}\left(t_{i}\right)-c_{12}^{12}\left(t_{i}\right) \\
\vdots \\
\rho_{12}^{1 K}\left(t_{i}\right)-c_{12}^{1 K}\left(t_{i}\right)
\end{array}\right)
\end{aligned}
$$

The minimization of Eq. (7) provides an optimized trade-off between minimizing the squared measurement residuals and minimizing the squared baseline length residuals. The first term of Eq. (7) can be further developed with Teunissen's orthogonal error decomposition [6] [7] [8], i.e.

$$
\begin{aligned}
\min _{\vec{b}_{12}, N_{12}}( & \left\|P_{H}\left(\Psi_{12}-H \vec{b}_{12}-A N_{12}\right)\right\|_{\Sigma_{\Psi_{12}}^{-1}}^{2} \\
& +\left\|P_{\bar{A}} P_{H}^{\perp}\left(\Psi_{12}-A N_{12}\right)\right\|_{\Sigma_{\Psi_{12}}^{-1}}^{2} \\
& \left.+\left(\left\|\vec{b}_{12}\right\|-\bar{l}_{12}\right)^{2} / \sigma_{\bar{l}_{12}}^{2}+\left\|P_{\bar{A}}^{\perp} P_{H}^{\perp} \Psi_{12}\right\|_{\Sigma_{\Psi_{12}}^{-1}}^{2}\right),
\end{aligned}
$$

where $P_{H}$ is the projector on the space of $H, P_{H}^{\perp}$ is the projector on its orthogonal complement, and $\bar{A}=P_{H}^{\perp} A$. The first and second term can be also expressed in terms of the float ambiguity solution $\hat{N}_{12}=\left(\bar{A}^{\mathrm{T}} \Sigma_{\Psi_{12}}^{-1} \bar{A}\right)^{-1} \bar{A}^{\mathrm{T}} \Sigma_{\Psi_{12}}^{-1} \Psi_{12}$, the fixed baseline estimate $\check{\vec{b}}_{12}=\left(H^{\mathrm{T}} \Sigma_{\Psi_{12}}^{-1} H\right)^{-1} H^{\mathrm{T}} \Sigma_{\Psi_{12}}^{-1}\left(\Psi_{12}-\right.$ $A \check{N}_{12}$ ), and the respective covariance matrices $\Sigma_{\hat{N}_{12}}$ and $\Sigma_{\breve{b}_{12}}$.

As the minimization over $N_{12}$ can not be solved in closed form, a search is required. We search for the best candidate $N_{12}$ inside a predefined search space volume $\chi^{2}$, i.e.

$\left\|\hat{N}_{12}-N_{12}\right\|_{\Sigma_{\hat{N}_{12}}^{-1}}^{2}+\left\|P_{\bar{A}}^{\perp} P_{H}^{\perp} \Psi_{12}\right\|_{\Sigma_{\Psi_{12}}^{-1}}^{2}$
$+\min _{\vec{b}_{12}}\left(\left\|\check{\vec{b}}_{12}\left(N_{12}\right)-\vec{b}_{12}\right\|_{\Sigma_{\tilde{b}_{12}^{-1}}^{-1}}^{2}+\left(\left\|\vec{b}_{12}\right\|-\bar{l}_{12}\right)^{2} / \sigma_{\bar{l}_{12}}^{2}\right) \leq \chi^{2}$.

We will now simplify the notation by omitting the index for the reference satellite in all DD measurements and ambiguities. Teunissen [6] used the conditional ambiguity estimate

$$
\begin{aligned}
\hat{N}_{12}^{k \mid 1, \ldots, k-1}= & \hat{N}_{12}^{k}-\sum_{j=1}^{k-1} \sigma_{\hat{N}_{12}^{k} \hat{N}_{12}^{j \mid 1, \ldots, j-1} / \sigma_{\hat{N}_{12}^{j \mid 1, \ldots, j-1}}^{2}} \\
& \cdot\left(\hat{N}_{12}^{j \mid 1, \ldots, j-1}-\left[\hat{N}_{12}^{j \mid 1, \ldots, j-1}\right]\right)
\end{aligned}
$$

to rewrite the first term of Eq. (10) as

$$
\left\|\hat{N}_{12}-N_{12}\right\|_{\Sigma_{\hat{N}_{12}}^{-1}}^{2}=\sum_{k=1}^{K} \frac{\left(\hat{N}_{12}^{k \mid 1, \ldots, k-1}-N_{12}^{k}\right)^{2}}{\sigma_{\hat{N}_{12}^{k \mid 1, \ldots, k-1}}^{2}},
$$


with $\sigma_{\hat{N}_{12}^{k \mid 1, \ldots, k-1}}^{2}$ being the variance of the conditional float ambiguity estimate. Replacing the squared ambiguity residuals of Inequality (10) by (12) and solving the squared inequality for $N_{12}^{k}$ yields a lower and an upper bound for the tree search:

$$
\begin{aligned}
& N_{12}^{k} \geq \hat{N}_{12}^{k \mid 1, \ldots, k-1}-\sqrt{\kappa^{k}} \cdot \sigma_{\hat{N}_{12}^{k \mid 1, \ldots, k-1}} \\
& N_{12}^{k} \leq \hat{N}_{12}^{k \mid 1, \ldots, k-1}+\sqrt{\kappa^{k}} \cdot \sigma_{\hat{N}_{12}^{k \mid 1, \ldots, k-1}}
\end{aligned}
$$

with the multiplier

$$
\begin{aligned}
\kappa^{k}= & \chi^{2}-\sum_{j=1}^{k-1} \frac{\left(\hat{N}_{12}^{j \mid 1, \ldots, j-1}-\check{N}_{12}^{j}\right)^{2}}{\sigma_{\hat{N}_{12}^{j \mid 1, \ldots, j-1}}^{2}}-\left\|P_{\bar{A}}^{\perp} P_{H}^{\perp} \Psi_{12}\right\|_{\Sigma_{\Psi_{12}}^{-1}}^{2} \\
& -\min _{\vec{b}_{12}}\left(\left\|\check{\vec{b}}_{12}\left(N_{12}\right)-\vec{b}_{12}\right\|_{\Sigma_{\check{b}_{12}}^{-1}}^{2}+\left(\left\|\vec{b}_{12}\right\|-\bar{l}_{12}\right)^{2} / \sigma_{\bar{l}_{12}}^{2}\right) .
\end{aligned}
$$

\section{HEADING DETERMINATION} WITH LOW-COST GPS RECEIVERS

In this section, we determine the heading of the vehicle based on the fixed baseline estimate. We assume that the baseline between the two GPS receivers is aligned with longitudinal axis of vehicle. The heading is $0^{\circ}$ in northern direction and counted clock-wise. In this case, the baseline vector is given by

$$
\vec{b}_{12}\left(t_{i}\right)=l \cdot\left(\begin{array}{c}
\sin \left(\psi\left(t_{i}\right)\right) \cos \left(\theta\left(t_{i}\right)\right) \\
\cos \left(\psi\left(t_{i}\right)\right) \cos \left(\theta\left(t_{i}\right)\right) \\
\sin \left(\theta\left(t_{i}\right)\right)
\end{array}\right)
$$

with the baseline length $l$, the heading $\psi$ and pitch $\theta$ of the vehicle. Thus, the heading and pitch angle estimates follow from the fixed baseline estimate by simple trigonometric properties as

$$
\begin{aligned}
& \hat{\psi}\left(t_{i}\right)=\operatorname{atan}\left(\left(\vec{b}_{12}\right)_{x}\left(t_{i}\right) /\left(\vec{b}_{12}\right)_{y}\left(t_{i}\right)\right) \\
& \hat{\theta}\left(t_{i}\right)=\operatorname{atan}\left(\left(\vec{b}_{12}\right)_{z}\left(t_{i}\right) / \sqrt{\left(\vec{b}_{12}\right)_{x}^{2}\left(t_{i}\right)+\left(\vec{b}_{12}\right)_{y}^{2}\left(t_{i}\right)}\right) .
\end{aligned}
$$

\section{CALIBRATION OF MAGNETIC FIELD SENSOR}

In this section, we provide a method for calibration of magnetic field sensors with two low-cost GPS receivers, i.e. we take the heading $\psi$ and pitch $\theta$ from the GPS carrier phase fixed attitude solution and consider it as true. Let $x$ be the vector of unknowns, i.e.

$$
x=\left(\Delta \theta, \Delta \psi, b_{x}, b_{y}, b_{z}, m_{\mathrm{N}}\left(t_{1}\right), \ldots, m_{\mathrm{N}}\left(t_{n}\right)\right)^{\mathrm{T}},
$$

then we obtain a least-squares estimate of $x$ by minimizing the squared magnetic flux residuals, i.e.

$$
\hat{x}=\arg \min _{x}\left\|m^{\mathrm{s}}-\bar{m}^{\mathrm{s}}(x)\right\|^{2},
$$

with $m^{\mathrm{s}}$ being the measured magnetic flux and $\bar{m}^{\mathrm{s}}$ being the computed magnetic flux measurement of all epochs, i.e.

$$
m^{\mathrm{s}}=\left(\begin{array}{c}
m^{\mathrm{s}}\left(t_{1}\right) \\
\vdots \\
m^{\mathrm{s}}\left(t_{n}\right)
\end{array}\right), \quad \bar{m}^{\mathrm{s}}=\left(\begin{array}{c}
\bar{m}^{\mathrm{s}}\left(t_{1}\right) \\
\vdots \\
\bar{m}^{\mathrm{s}}\left(t_{n}\right)
\end{array}\right) \text {, }
$$

where $m^{\mathrm{s}}\left(t_{i}\right)$ and $\bar{m}^{\mathrm{s}}\left(t_{i}\right)$ are given by

$$
\begin{aligned}
& m^{\mathrm{s}}\left(t_{i}\right)=\left(m_{x}^{\mathrm{s}}\left(t_{i}\right), m_{y}^{\mathrm{s}}\left(t_{i}\right), m_{z}^{\mathrm{s}}\left(t_{i}\right)\right)^{\mathrm{T}} \\
& \bar{m}^{\mathrm{s}}\left(t_{i}\right) \\
& \quad=\left(\begin{array}{r}
\cos \left(\theta\left(t_{i}\right)+\Delta \theta\right) \sin \left(\psi\left(t_{i}\right)+\Delta \psi\right) m_{\mathrm{N}}\left(t_{i}\right)+b_{x} \\
\cos \left(\psi\left(t_{i}\right)+\Delta \psi\right) m_{\mathrm{N}}\left(t_{i}\right)+b_{y} \\
\sin \left(\theta\left(t_{i}\right)+\Delta \theta\right) \sin \left(\psi\left(t_{i}\right)+\Delta \psi\right) m_{\mathrm{N}}\left(t_{i}\right)+b_{z}
\end{array}\right) .
\end{aligned}
$$

Obviously, there is some change in attitude required to separate the misalignment errors, magnetic flux and biases and, thus, to perform the calibration.

As Eq. (19) represents a non-linear least-squares problem, we perform a linearization and solve it iteratively with the Gauss-Newton method. A Taylor series expansion is performed up to order 1 around the state estimate $\hat{x}^{(j-1)}$ of the previous iteration $j-1$. The linearized $m^{\mathrm{s}}$ at iteration $j$ is modeled as

$$
m^{\mathrm{s}}=\left.\bar{m}^{\mathrm{s}}\right|_{x=x^{(j-1)}}+\left.J\right|_{x=x^{(j-1)}} \cdot\left(x-x^{(j-1)}\right)+\eta_{m},
$$

with the Jacobian matrix $J=\left(J_{1}, J_{2}\right)$. It consists of two parts, i.e. of one part including the partial derivatives w.r.t. the misalignment errors and biases given by

$$
J_{1}=\left(\begin{array}{lllll}
\frac{\partial m^{\mathrm{s}}}{\partial \Delta \theta} & \frac{\partial m^{\mathrm{s}}}{\partial \Delta \psi} & \frac{\partial m^{\mathrm{s}}}{\partial b_{x}} & \frac{\partial m^{\mathrm{s}}}{\partial b_{y}} & \frac{\partial m^{\mathrm{s}}}{\partial b_{z}}
\end{array}\right),
$$

and of a second part including the partial derivatives w.r.t. the magnetic flux for every epoch, i.e.

$$
J_{2}=\left(\begin{array}{ccc}
\frac{\partial m^{\mathrm{s}}}{\partial m_{\mathrm{N}}\left(t_{1}\right)} & \cdots & \frac{\partial m^{\mathrm{s}}}{\partial m_{\mathrm{N}}\left(t_{n}\right)}
\end{array}\right) .
$$

The partial derivatives can be easily obtained from Eq. (21), e.g. the entries of the first column and first three rows of $J_{1}$ are given by

$\frac{\partial m^{\mathrm{s}}\left(t_{1}\right)}{\partial \Delta \theta}=\left(\begin{array}{r}-\sin \left(\theta\left(t_{1}\right)+\Delta \theta\right) \sin \left(\psi\left(t_{1}\right)+\Delta \psi\right) m_{\mathrm{N}}\left(t_{1}\right) \\ 0 \\ \cos \left(\theta\left(t_{1}\right)+\Delta \theta\right) \sin \left(\psi\left(t_{1}\right)+\Delta \psi\right) m_{\mathrm{N}}\left(t_{1}\right)\end{array}\right)$

The least-squares estimate of the state parameters at the $j$-th iteration is then given by

$$
\begin{aligned}
\hat{x}^{(j)}= & \hat{x}^{(j-1)} \\
& +\left.\left(\left.\left.J\right|_{x=\hat{x}^{(j-1)}} ^{\mathrm{T}} J\right|_{x=\hat{x}^{(j-1)}}\right)^{-1} J\right|_{x=\hat{x}^{(j-1)}} ^{\mathrm{T}} \cdot\left(m^{\mathrm{s}}-\bar{m} \mid\right) .
\end{aligned}
$$

Obviously, the iterative Gauss-Newton method needs (as every iterative method) an initialization of the state vector. We assume only for the initialization that the biases $b_{x}, b_{y}$ and $b_{z}$ are zero and that there are no misalignment errors $\Delta \theta$ and $\Delta \psi$. In this case, $m_{\mathrm{N}}\left(t_{i}\right)$ can be derived directly from the measurements as

$$
m_{\mathrm{N}}^{(0)}\left(t_{i}\right)=\sqrt{\left(m_{x}^{\mathrm{s}}\left(t_{i}\right)\right)^{2}+\left(m_{y}^{\mathrm{s}}\left(t_{i}\right)\right)^{2}+\left(m_{z}^{\mathrm{s}}\left(t_{i}\right)\right)^{2}} .
$$

Thus, the state vector can be initialized with

$$
\hat{x}^{(0)}=\left(0^{1 \times 2+3}, m_{\mathrm{N}}^{(0)}\left(t_{1}\right), \ldots, m_{\mathrm{N}}^{(0)}\left(t_{n}\right)\right)^{\mathrm{T}} .
$$




\section{Measurement Results}

In this section, we verify the proposed calibration method with pseudorange and carrier phase measurements from two $5 \mathrm{~Hz}$ u-blox LEA 6T GPS receivers and three-dimensional magnetic flux measurements from one CMPS10 sensor. The GPS receivers were connected to two L1 patch antennas that were mounted on the roof of the vehicle together with the CMPS10 sensor. The baseline length between both GPS receivers was $1.4 \mathrm{~m}$. Fig. 1 shows the track of the vehicle during the calibration at Nymphenburg Castle, Munich, Germany.

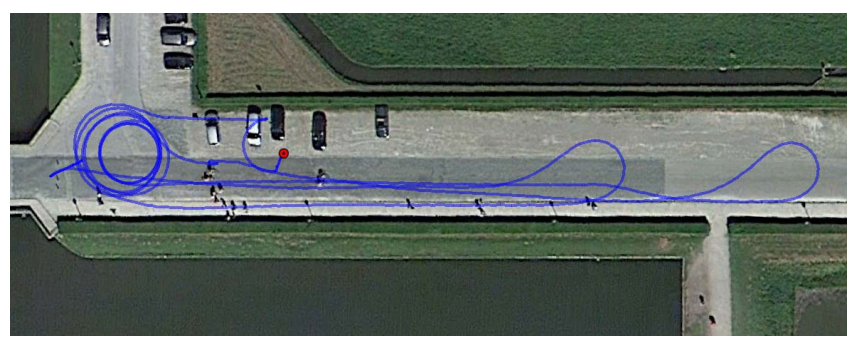

Fig. 1. Track of vehicle: The driving of numerous circles is ideally suited for calibration of magnetometers. The starting point of the track is marked by a red point.

\section{A. Calibration of magnetometers}

In this subsection, we verify the proposed measurement model for the CMPS10 magnetometer with real measurements, apply the proposed GPS-based calibration method of magnetometers, and show the achievable accuracy of the magneticfield based heading. Fig. 2-4 show a comparison of the measured and estimated magnetic flux measurements based on Eq. (21). The estimated magnetic flux closely follows the track of the measured magnetic flux, which indicates the correctness of the measurement model. The $z$-component of the magnetic flux is constant for most of the time, and only shows some oscillations during the sharp turns.

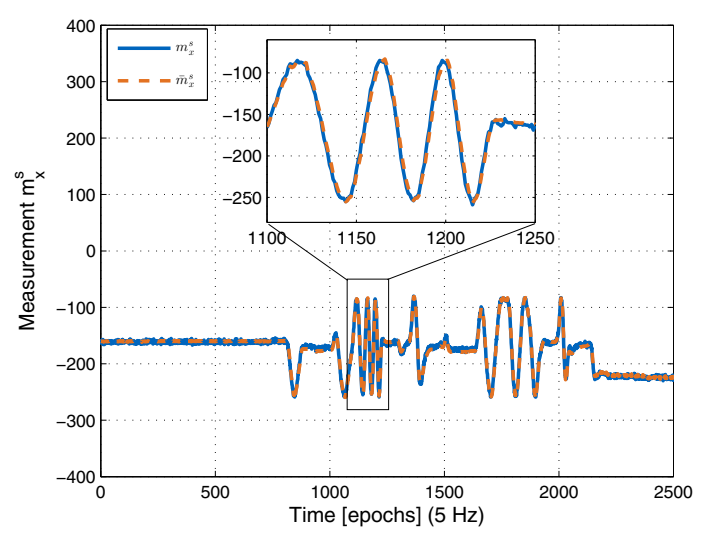

Fig. 2. Comparison of measured and estimated magnetic flux in $\mathrm{x}$-direction The estimated magnetic flux closely follows the track of the measured magnetic flux, which indicates the correctness of the measurement model.

Fig. 5 includes a comparison of the GPS-based heading and of the magnetic field-based heading after calibration. The noise

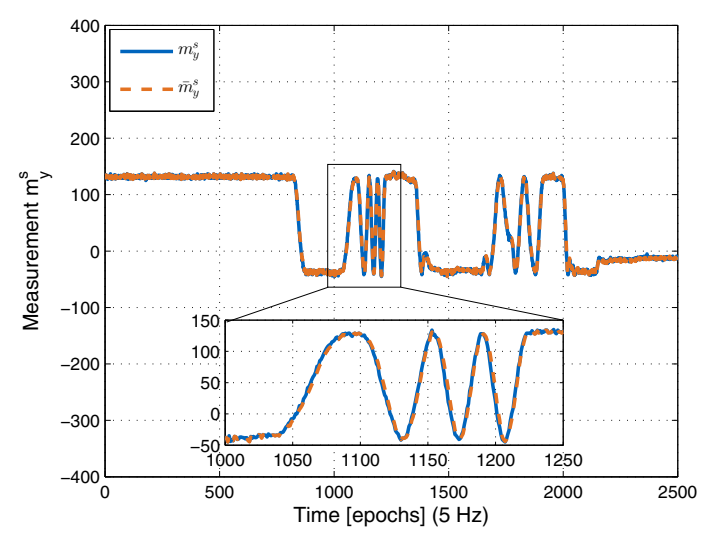

Fig. 3. Comparison of measured and estimated magnetic flux in y-direction: The estimated magnetic flux follows again the track of the measured magnetic flux, which indicates the correctness of the measurement model.

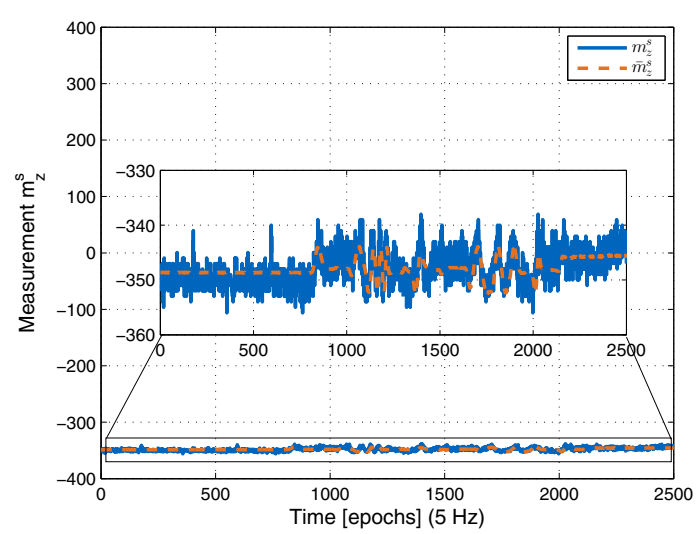

Fig. 4. Comparison of measured and estimated magnetic flux in z-direction The $z$-component of the magnetic flux is constant for most of the time. During the sharp turns, the magnetic flux oscillates to a certain extent. The noise level of the measured magnetic flux is in the order of a few degrees.

level of the magnetic field based heading is in the order of only a few degrees, which makes the magnetometer very attractive to support GPS integer ambiguity resolution. The noise level of the GPS based heading is in the order of only $0.2^{\circ}$ and, thus, can be used to analyze the performance of the magnetometer.

Fig. 6 shows the difference between the GPS based and the magnetic field based heading estimates. One can observe an almost zero-mean noise of a few degrees within the first 800 epochs. The offset increases around epoch 1200 to $15^{\circ}$, which indicates some local magnetic disturbances. In total, the difference is less than $5^{\circ}$ in $92.6 \%$ and less than $10^{\circ}$ in $99.5 \%$ of all epochs.

\section{B. Heading determination with calibrated magnetometers}

In this section, we use the calibration correction for testing the performance of the magnetic field based heading with a longer data set (8500 epochs). Fig. 7 shows the obtained heading estimate in comparison to the GPS-based heading. The first enlarged area shows that the heading can deviate by 


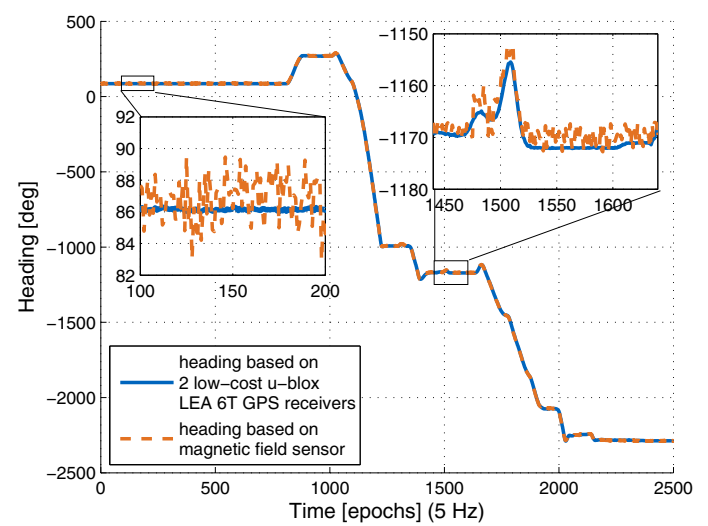

Fig. 5. Comparison of GPS-based and magnetic-flux based heading estimates: The noise level of the magnetic-field based heading is in the order of a few degrees. The GPS-based heading shows a noise level in the order of only $0.2^{\circ}$ and, thus, serves as a reference.

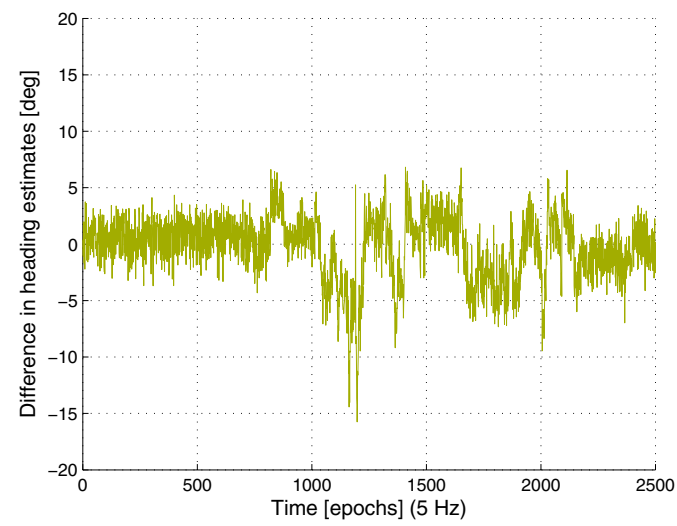

Fig. 6. Difference in heading estimates between GPS and magnetometer: The difference is less than $5^{\circ}$ in $92.6 \%$ and less than $10^{\circ}$ in $99.5 \%$ of all epochs. Thus, the magnetometer can fasten ambiguity resolution.

up to $40^{\circ}$ in case of dynamic disturbances (e.g. crossing below tram power cables), while the second enlarged area shows a comparison of both noise levels: The noise level is in the order of $0.2^{\circ}$ for GPS and in the order of a few degrees for the magnetometer. Fig. 8 shows the difference between both heading estimates: The offset remains less than $5^{\circ}$ in $78.1 \%$ and less than $10^{\circ}$ in $95.4 \%$ of all epochs.

\section{CONCLUSION}

In this paper, we presented a method to calibrate magnetometers with the help of two GPS receivers. The method first determines the attitude with GPS which implies a tree search of the carrier phase integer ambiguities. Subsequently, the misalignment errors, measurement biases and magnetic flux including static disturbances are computed with the iterative Gauss-Newton method.

The proposed method was tested with two low-cost GPS receivers and a magnetic field sensor in a test drive. The calibrated heading differed by less than $10^{\circ}$ in $99.5 \%$ of all epochs from the GPS-based heading.

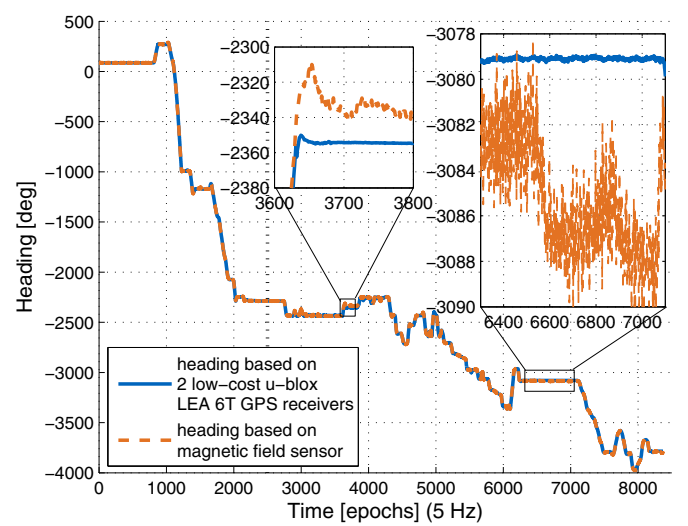

Fig. 7. Challenges of heading determination with calibrated magnetometers: The first enlarged area shows heading errors of up to $40^{\circ}$ during dynamic disturbances, while the second enlarged area shows that the noise level is significantly larger than in case of GPS.

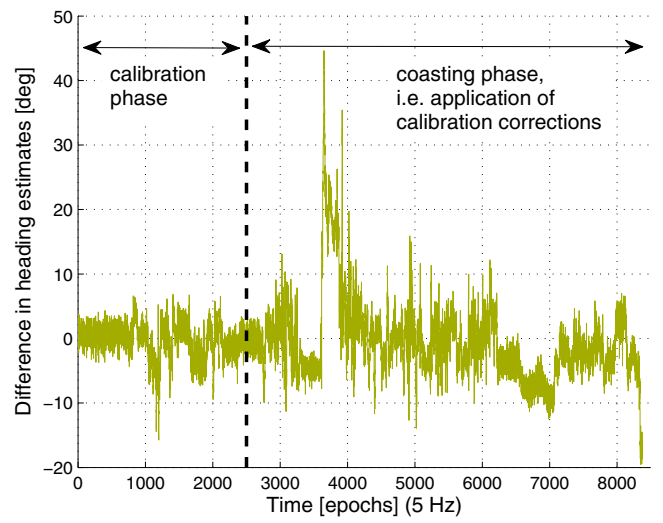

Fig. 8. Difference in heading estimate based on two u-blox LEA 6T GPS receivers and the heading based on the calibrated CMPS10 magnetometer: The offset remains less than $10^{\circ}$ in $95.4 \%$ of all epochs.

\section{REFERENCES}

[1] J. Crassidis, K.-L. Lai and R. Harman, Real-time attitude-independent three axis magnetometer calibration, J. of Guidance, Control, and Dynamics, vol. 28, no. 1, pp. 115-120, 2005.

[2] M. Psiaki, F. Martel and P. Pal, Three-axis attitude determination via Kalman filtering of magnetometer data, Journal of Guidance, Control, and Dynamics, vol. 13, no. 3, pp. 506-514, 1990.

[3] P. Henkel and J. Kiam, Maximum A Posteriori Probability Estimation of Integer Ambiguities and Baseline, Proc. of 55-th IEEE Symp. ELMAR, pp. 353-356, Zadar, Croatia, Sep. 2013.

[4] P. Henkel and C. Günther, Attitude determination with low-cost GPS/ INS, Proc. of the 26-th Intern. Techn. Meeting of the Satellite Division of the Institute of Navigation (ION GNSS+), Nashville, TN, USA, pp. 2015-2023, Sep. 2013.

[5] P. Henkel and N. Oku, Cycle slip detection and correction for heading determination with low-cost GPS/ INS receivers, IAG Proc. of VIII Hotine Marussi Symposium, Rome, Italy, Jun. 2013.

[6] P. Teunissen, The least-squares ambiguity decorrelation adjustment: a method for fast GPS ambiguity estimation, J. of Geodesy, vol. 70, pp. 65-82, 1995.

[7] P. Teunissen, The LAMBDA method for the GNSS compass, Artificial Satellites, vol. 41(3), pp. 89-103, 2006

[8] P. Teunissen, Integer least-squares theory for the GNSS compass, J. of Geodesy, vol. 84, pp. 433-447, 2010. 